\title{
O cigarro, o narguilé e a doença periodontal
}

\author{
Cigarette, narguilé and periodontal disease \\ El cigarro, el narguilé y la enfermedad periodontal
}

Evandro Franco da Rocha ${ }^{1 *}$, Fernanda da Silva ${ }^{1}$, Lavini da Rosa Agostini ${ }^{1}$, Valeria Campanelli Franco da Rocha ${ }^{2}$.

\section{RESUMO}

Objetivo: Revisar a associação entre o cigarro, o narguilé e o desenvolvimento da doença periodontal. Métodos: Foram realizadas pesquisas em revistas, livros, e artigos científicos encontrados através dos sites de buscas PubMed, Scielo, Portal Capes e Google Acadêmico. Resultados: Atualmente a prevalência de fumantes é de 1,3 bilhões no mundo, sendo o tabagismo considerado uma doença epidemiológica, pois causa dependência pelas substâncias presentes em sua composição. Estas substâncias, além da dependência, causam danos à saúde bucal, atuando como fator modificador no desenvolvimento da doença periodontal, por interferência local e sistêmica, além de influenciar negativamente no resultado do seu tratamento. Considerações finais: $O$ uso do tabaco independente da sua forma de apresentação, está relacionado diretamente com o desenvolvimento da doença periodontal. Novas políticas de regulamentação, ações de promoção e prevenção para saúde, devem ser implementadas, principalmente levando em a crescente prevalência do narguilé entre adultos jovens e adolescentes, fora das regiões em que era tradicionalmente conhecido.

Palavras-chave: Doença Periodontal, Tabagismo, Narguilé.

\begin{abstract}
Objective: Review the association between cigarette, narguile and the development of periodontal disease. Methods: Researches were conducted in magazines, books, and scientific articles found through the PubMed, Scielo, Capes Portal and Google Scholar sites. Results: Currently the prevalence of smokers is 1.3 billion in the world, and smoking is considered an epidemiological disease, because it causes dependence on the substances present in its composition. These substances, in addition to dependence, cause damage to oral health, acting as a modifying factor in the development of periodontal disease, due to local and systemic interference, as well as negatively influencing the outcome of their treatment. Final considerations: Tobacco use regardless of its presentation is directly related to the development of periodontal disease. New regulatory policies, health promotion and prevention actions should be implemented, mainly leading to the increasing prevalence of narghile among young adults and adolescents, outside the regions where it was traditionally known.
\end{abstract}

Key words: Periodontal Disease, Smoking, Narghile.

\section{RESUMEN}

Objetivo: Revisar la asociación entre el cigarrillo, el narguile y el desarrollo de la enfermedad periodontal. Métodos: Se realizaron investigaciones en revistas, libros, y artículos científicos encontrados a través de los sitios de búsqueda PubMed, Scielo, Portal Capes y Google Académico. Resultados: Actualmente la

${ }^{1}$ Universidade Regional de Blumenau (FURB), Blumenau SC. *E-mail: efrocha@furb.br 
prevalencia de fumadores es de 1.300 millones en el mundo, siendo el tabaquismo considerado una enfermedad epidemiológica, pues causa dependencia por las sustancias presentes en su composición. Estas sustancias, además de la dependencia, causan daños a la salud bucal, actuando como factor modificador en el desarrollo de la enfermedad periodontal, por interferencia local y sistémica, además de influenciar negativamente en el resultado de su tratamiento. Consideraciones finales: El uso del tabaco independiente de su forma de presentación, está relacionado directamente con el desarrollo de la enfermedad periodontal. Las nuevas políticas de regulación, acciones de promoción y prevención para la salud, deben ser implementadas, principalmente llevando en la creciente prevalencia del narguile entre adultos jóvenes y adolescentes, fuera de las regiones en que era tradicionalmente conocido.

Palabras clave: Enfermedad Periodontal, Tabaquismo, Narguile.

\section{INTRODUÇÃO}

Atualmente a prevalência de fumantes no mundo é de 1,3 bilhões, sendo que no Brasil esse índice chega a 27,9 milhões (WHO, 2017).

O tabagismo pode ser considerado como uma doença epidemiológica, pois causa dependência física, comportamental e psicológica e isso assemelha-se ao que ocorre quando se faz o uso de drogas como cocaína, heroína e álcool, além de estar relacionado com mais de 50 tipos de doenças, alto índice de mortalidade, e prejuízos sociais e econômicos (INCA, 2013).

Apesar de tantas informações e campanhas de prevenção no Brasil e no mundo, a incidência de indivíduos que fazem uso do tabaco ainda é grande. Desta forma, explorar e discutir os efeitos do tabaco, independente da forma de sua utilização: seco, picado, enrolado em papel, em palha, de corda, charuto, narguilé, cachimbo, entre outros, se faz necessário (CARRANZA FA et al. 2016).

A doença periodontal (DP) é caracterizada por uma inflamação no tecido periodontal de suporte causada por uma infecção bacteriana, e possui como fator etiológico principal o biofilme dental, que é um acúmulo de bactérias da microbiota bucal sobre a superfície dos dentes; e que é o fator determinante para que ocorra a cárie e a doença periodontal. Esta inflamação pode ser reversível (gengivite) ou irreversível, quando ocorre perda de inserção conjuntiva e óssea - periodontite (SOUZA AB, et al. 2010, AKCALI A e LANG NP 2018).

Os resultados do Projeto SB Brasil 2010 indicam que o percentual de indivíduos sem nenhum problema periodontal foi de $63 \%$ para a idade de 12 anos, $50,9 \%$ para a faixa de 15 a 19 anos, $17,8 \%$ para os adultos de 35 a 44 anos e somente $1,8 \%$ nos idosos de 65 a 74 anos. (MINISTÉRIO DA SAÚDE, 2012.)

Por seu caráter multifatorial, apesar de ser o biofilme (placa bacteriana) o fator etiológico principal, vários fatores podem estar associados ao desenvolvimento desta doença, como sistêmicos, hereditários, locais, alcoolismo e uso de tabaco (CARRANZA FA et al. 2016), sendo o tratamento inicialmente não cirúrgico, através de raspagem e alisamento radicular, e controle dos fatores modificadores (locais ou sistêmicos), podendo ser complementado por terapia cirúrgica (CAFFESSE RG e ECHEVERRÍA JJ, 2019).

O fumo é um dos fatores coadjuvantes mais significativos no desenvolvimento de doenças periodontais (DPs) por sua interferência local e sistêmica no desencadeamento e na progressão da periodontite, pois o tabaco além de ser um fator predisponente à doença periodontal, também pode influenciar no resultado do seu tratamento, sabendo que as substâncias que estão relacionadas ao tabagismo agem como vasoconstritores causando danos ao tecido periodontal, o que leva a progressão da doença (CARVALHO AE et al. 2008).

Neste contexto, esta revisão de literatura visa reforçar o conhecimento entre a associação do cigarro, o narguilé e o desenvolvimento e posterior progressão da DP.

\section{REVISÃO BIBLIOGRÁFICA}

REAS/EJCH | Vol. Sup.28 | e784 | DOI: https://doi.org/10.25248/reas.e784.2019 Página 2 de $\mathbf{6}$ 
Sabe-se que nos últimos anos o uso do tabaco em suas diversas formas vem crescendo significativamente. Apesar de muitos de seus efeitos citotóxicos já serem conhecidos pela maioria dos indivíduos que fazem uso do mesmo, também pode-se afirmar que há uma íntima relação entre o tabaco e a DP, o que causa efeitos negativos à saúde do periodonto, como afirmam os autores Salum AW et al. (2007) e Dinelli W et al. (2009).

Apesar de o cigarro ainda ser o tipo de tabagismo mais comum atualmente, pode-se afirmar que o uso do narguilé vem crescendo alarmantemente nos últimos anos, principalmente entre os jovens e estudantes universitários, já que atinge cerca de 212 mil brasileiros acima de 18 anos (IBGE, 2015).

Apesar de sua prática ter se tornado muito comum atualmente, os usuários que fazem o uso do narguilé ainda não possuem muito conhecimento dos malefícios que esta prática pode causar, tanto a saúde periodontal quanto a saúde geral, já que os mesmos enganam-se achando que os efeitos do narguilé não são tão nocivos quanto aos efeitos do cigarro. É sabido que o narguilé usa como fonte de calor a combustão do carvão, e o mesmo possui vários produtos tóxicos, dentre eles o monóxido de carbono que está presente em altas concentrações, além de que o número de tragadas feitas pelo usuário influencia diretamente na toxicidade a que o indivíduo é exposto (IARC, 2012).

A associação entre tabagismo e periodontite é certa, segundo Leite FRM et al, 2018. Os autores pesquisaram estudos prospectivos longitudinais abordando a associação entre tabagismo e incidência e progressão da periodontite. As vias pelas quais o fumo do tabaco afeta a incidência e a progressão da periodontite permanecem incertas; entretanto, alguns mecanismos potenciais foram hipotetizados. Eles incluem o efeito do tabagismo sobre a composição da microbiota, sobre a resposta imune e sobre a capacidade de cura do periodonto. Em conjunto, esses achados apoiam a associação entre tabagismo e incidência e progressão da periodontite. Como o tabagismo e a periodontite ainda são grandes problemas de saúde pública, mais investimentos em iniciativas de pesquisa com o objetivo de investigar as interações entre o tabagismo e outras causas potenciais de periodontite precisam se tornar uma prioridade.

O uso de várias formas de tabaco é um dos mais importantes fatores de risco evitáveis para a incidência e progressão da doença periodontal. $O$ uso de tabaco afeta negativamente os resultados do tratamento tanto para doenças e condições periodontais como para implantes dentários. Estudos têm demonstrado taxas significativamente mais elevados de agentes patogénicos periodontais em fumantes (RYDER MI et al. 2018).

De acordo com Jhonson GK e Guthmiller JM (2007), Carvalho AE et al. (2008), Underner M et al.(2009) e Douglass C (2006), o tabaco possui influência direta no sucesso ou insucesso da terapia periodontal, já que sabe-se que além de afetar os tecidos do periodonto, a nicotina presente no tabaco irá causar vasoconstrição, ocultando os sinais da inflamação e assim dificultando seu correto diagnóstico, além de aumentar as chances de se ter resposta negativa à terapia periodontal, contribuindo para uma possível recorrência da doença.

O papel do tabagismo na promoção do crescimento de bactérias patogênicas orais pode estar em alterar diretamente $\mathrm{o}$ ambiente em que os microrganismos crescem ou prejudicando a resposta protetora do hospedeiro, incluindo a modulação da resposta inflamatória, que por si só alteraria indiretamente o ambiente do biofilme e permitiria um maior crescimento de organismos patogênicos (RYDER MI et al 2018).

Os microrganismos que colonizam nosso corpo se adaptaram e evoluíram durante milhões de anos, e portanto, na maior parte do tempo, convivem em harmonia e equilíbrio conosco (microbioma). Porém microrganismos patógenos periodontais, expostos à nicotina e ao extrato da fumaça do tabaco, sofrem alterações funcionais que sustentam os achados clínicos relacionados à disbiose (estado alterado da ecologia microbiana) do microbioma subgengival. A disbiose do microbioma periodontal foi apresentada em fumantes independentemente de sua condição periodontal (saudável, gengivite ou periodontite) e permaneceu significativa apenas em fumantes, mesmo após a resolução da gengivite e da redução dos sinais clínicos de periodontite. A cessação do tabagismo em pacientes com periodontite é claramente benéfico para promover uma comunidade microbiana subgengival compatível com a saúde (HANIOKA T et al 2019).

O uso de tabaco é um fator de risco principal para a periodontite crônica. $O$ tabagismo leva à supressão da inflamação gengival, manifestada como angiogênese reduzida e comprometimento da resposta de 
sangramento à placa, mas promove simultaneamente a destruição do tecido periodontal em relação aos não fumantes. A resposta ao sangramento se recupera rapidamente após a cessação do tabagismo. Os mecanismos subjacentes à supressão vascular em usuários de cigarro ainda precisam ser totalmente elucidados. Embora possa haver um elemento genético para a suscetibilidade, o uso do tabaco influencia profundamente os mediadores biológicos que controlam as respostas angiogênicas e hemorrágicas aos desafios bacterianos; promove disbiose bacteriana oral; compromete a função celular inata; e promove um desequilíbrio protease-antiprotease nos tecidos gengivais. Pode ser possível que abordagens preventivas e terapêuticas específicas para o controle da periodontite crônica, e outras doenças orais destrutivas induzidas por placa, precisem ser adaptadas para fumantes (BUDUNELI N e SCOTT DA 2018).

Estudos recentes abordam o efeito do tabagismo sobre fatores moleculares e genéticos na doença periodontal. A maioria confirma o fato de que o fumo do tabaco modula a destruição do periodonto através de diferentes vias: sistema imune microcirculatório e do hospedeiro, tecido conjuntivo e metabolismo ósseo (OJIMA M e HANIOKA T 2010).

Também parece haver uma relação dose-efeito entre o tabagismo e a gravidade do desfecho da doença periodontal. Vários estudos transversais foram realizados para estudar esta relação. $O$ tabagismo pesado foi consistentemente associado a doença periodontal mais severa do que o tabagismo leve, e o número de anos de tabagismo foi significativamente associado à perda de dentes e doença periodontal, independentemente de outros fatores sociais e comportamentais (BERGSTRÖM J et al 2000, ALBANDAR JM, 2002). Dentro desta perspectiva de dose dependência, relacionamos no Quadro 1, as características comparativas de uso entre o cigarro tradicional e o narguilé, demonstrando que em uma sessão típica do narguilé, que leva cerca de uma hora aproximadamente, o usuário é exposto a doses elevadas de substâncias tóxicas que comparadas ao cigarro, equivalendo a fumar cerca de dez cigarros comuns. além de ocorrer a liberação de diversas substâncias citotóxicas causada pela fumaça da queima do carvão presente neste artefato (IARC, 2012).

Quadro 1 - Quadro comparativo entre cigarro e narguilé.

\begin{tabular}{|c|c|}
\hline CIGARRO & NARGUILÉ \\
\hline Possui filtro; & Não possui filtro; \\
\hline $\begin{array}{l}\text { Possui uma quantidade menor de monóxido de } \\
\text { carbono quando comparado ao narguilé; } \\
\text { (VIEGAS CAA, 2008). }\end{array}$ & $\begin{array}{l}\text { Quantidade de monóxido de carbono é de } 10 \text { a } \\
30 \text { vezes maior que o cigarro (por não possuir } \\
\text { filtro e necessitar da queima de carvão); } \\
\text { (VIEGAS CAA, 2008). }\end{array}$ \\
\hline $\begin{array}{l}\text { O volume de tragadas do cigarro alcança } 40 \text { a } 75 \\
\text { ml entre } 5 \text { a } 7 \text { minutos; (VIEGAS CAA, 2008). }\end{array}$ & $\begin{array}{l}\text { O volume de tragadas do narguilé pode chegar a } \\
1000 \mathrm{ml} \text { em uma sessão de uma hora; (VIEGAS } \\
\text { CAA, 2008). }\end{array}$ \\
\hline \multirow[t]{4}{*}{$\begin{array}{l}\text { Geralmente um cigarro leva de } 5 \text { a } 7 \text { minutos para } \\
\text { acabar e propicia de } 8 \text { a } 12 \text { baforadas; (VIEGAS } \\
\text { CAA, 2008). }\end{array}$} & $\begin{array}{l}\text { Geralmente um encontro onde há narguilé dura } \\
\text { de } 20 \text { a } 80 \text { minutos e pode render entre } 50 \text { a } 200 \\
\text { baforadas; (VIEGAS CAA, 2008). }\end{array}$ \\
\hline & $\begin{array}{l}\text { Sessão de narguilé de } 80 \text { minutos equivale a } \\
\text { fumaça de aproximadamente } 100 \text { cigarros; } \\
\text { (VIEGAS CAA, 2008). }\end{array}$ \\
\hline & $\begin{array}{l}\text { Após uma sessão de } 45 \text { minutos ocorre } 0 \\
\text { aumento dos batimentos cardíacos; }\end{array}$ \\
\hline & $\begin{array}{l}\text { O uso a longo prazo pode causar várias doenças } \\
\text { como câncer de pulmão, de boca, doenças } \\
\text { cardíacas, respiratórias e aterosclerose; (INCA, } \\
\text { 2013). }\end{array}$ \\
\hline $\begin{array}{l}\text { Geralmente de uso individual, não compartilhado. } \\
\text { (INCA, 2017). }\end{array}$ & $\begin{array}{l}\text { Pode causar doenças infectocontagiosas como } \\
\text { herpes, hepatite } \mathrm{C} \text { e tuberculose devido ao } \\
\text { compartilhamento do bocal pelos usuários. } \\
\text { (RIBEIRO M, CRUZ RC, 2016). }\end{array}$ \\
\hline $\begin{array}{l}\begin{array}{l}\text { Conteúdo de nicotina estimado entre } 1-3 \% \\
\text { (VIEGAS CAA, 2008). }\end{array} \\
\end{array}$ & $\begin{array}{l}\text { Conteúdo de nicotina estimado entre } 2-4 \% \\
\text { (VIEGAS CAA, 2008). }\end{array}$ \\
\hline
\end{tabular}

Fonte: Dados de pesquisa, 2019. 
Apesar de ser uma prática muito comum atualmente, os usuários não têm conhecimento sobre os malefícios e riscos que o narguilé pode causar, tanto a saúde bucal quanto a saúde geral, já que é sabido que seus efeitos podem ser tão nocivos quanto os efeitos do cigarro (KHEMISS M et al. 2016).

O uso de narguilé entre adolescentes está crescendo constantemente devido seu poder socializador, pois os mesmos utilizam o narguilé quando estão reunidos em grupos, por terem a aprovação dos pais que na maioria dos casos não tem conhecimento sobre os riscos que o uso do narguilé pode causar a saúde, além do fácil acesso para adquirir estes tipos de produtos (SAPIENZA G e SCARINCI IC, 2018).

Apesar da prevalência do uso de narguilé ser menor quando comparado ao uso de outros tipos de tabaco, é necessário ter um conhecimento sistematizado, para realizar a conscientização dos usuários, principalmente para o público jovem (RIBEIRO M e CRUZ RC, 2016).

Com o aumento de usuários de narguilé, ocorre a necessidade de implantação de políticas de regulamentação para venda e uso do mesmo, além de ações de promoção para alertar os usuários sobre os riscos e malefícios que este artefato pode causar (SAPIENZA G e SCARINCI IC, 2018).

A regularização e a implantação de políticas públicas devem ser analisadas e estabelecidas, dando prioridade a ações educativas que irão alertar os riscos que o uso do narguilé pode causar a saúde (RIBEIRO M e CRUZ RC, 2016).

\section{CONSIDERAÇÕES FINAIS}

O uso do tabaco, independentemente de sua forma de apresentação, está relacionado diretamente com o desenvolvimento e progressão da doença periodontal, além de outros inúmeros tipos de doenças, tendo um efeito dose dependente. Sabendo que o número dos usuários de narguilé entre adultos jovens e adolescentes, fora das regiões em que era tradicionalmente conhecido, vem crescendo drasticamente nos últimos anos, ocorre a necessidade de novas políticas de regulamentação para uso e venda desses produtos. Além disso, são necessárias ações de promoção e prevenção de saúde para a conscientização dos usuários, principalmente entre os jovens, alertando para os riscos que o uso de tabaco e seus derivados podem causar à saúde periodontal.

\section{REFERÊNCIAS}

1. AKCALI A, LANG NP. Dental calculus: the calcified biofilm and its role in disease development. Periodontology 2000 , 2018; 76(1):109-115.

2. ALBANDAR JM. Global risk factors and risk indicators for periodontal diseases. Periodontology 2000, 2002; 29:177206.

3. BERGSTRÖM J. et al Exposure to tobacco smoking and periodontal health. Journal of Clinical Periodontology, 27(1): 61-68.

4. BUDUNELI N, SCOTT DA. Tobacco-induced suppression of the vascular response to dental plaque. Molecular Oral Microbiology, 2018; 33(4):271-282.

5. CAFFESSE RG, ECHEVERRÍA JJ Treatment trends in periodontics. Periodontology 2000, 2019; 79(1):7-14.

6. CARRANZA FA, et al. Periodontia clínica 12.ed., Rio de Janeiro, Elsevier, 2016.

7. CARVALHO AE et al. A influência do tabagismo na doença periodontal: Revisão de literatura. Revista Virtual de Odontologia, 2008, 2(5):7-12.

8. DINELLI, W et al. Análise do índice de placa gengival e higiene bucal de pacientes em relação ao tabagismo. RGO Revista Gaúcha de Odontologia, 2009; 56(4):46-52.

9. DOUGLASS C Uso de produtos de tabaco prejudica a saúde periodontal. Prev News, 2006; 15(2):1-3.

10. HANIOKA T, et al. Smoking and periodontal microorganisms. Japanese Dental Science Review, 2019; 55(1):88-94.

11. IARC Personal habits and indoor combustions. volume $100 \mathrm{E}$ a review of human carcinogens. IARC Monographs on the Carcinogenic Risk of Chemicals to Humans, International Agency for Research on Cancer, Lyon, 2012.

12. IBGE Pesquisa nacional de saúde: 2013: acesso e utilização dos serviços de saúde, acidentes e violências: Brasil, grandes regiões e unidades da federação, Instituto Brasileiro de Geografia e Estatística, Rio de Janeiro, 2015.

13. INCA Instituto Nacional do Câncer. Uso de narguilé: efeitos sobre a saúde, necessidades de pesquisa e ações recomendadas para legisladores. 2. ed., Ministério da Saúde, Rio de Janeiro, 2017. 
14. INCA. Instituto Nacional do Câncer. Programa Nacional de Controle do Tabagismo. Ministério da Saúde, Rio de Janeiro, 2013.

15. JHONSON GK, GUTHMILLER JM The impact of cigarette smoking on periodontal disease and treatment. Journal Periodontology, 2007; 4(4):178-194.

16. KHEMISS $M$ et al, Periodontal bone height of exclusive narghile smokers compared with exclusive cigarette smokers, Libyan Journal of Medicine, 2016; 11(1):1-9.

17. LEITE FRM, et al Effect of Smoking on Periodontitis: A Systematic Review and Meta-regression. The American Journal of Preventive Medicine, 2018; 54(6):831-841.

18. MINISTÉRIO DA SAÚDE Secretaria de Atenção à Saúde. SB BRASIL 2010 Pesquisa Nacional de Saúde Bucal Resultados Principais. Brasília, Ministério da Saúde, 2012.

19. OJIMA M, HANIOKA T Destructive effects of smoking on molecular and genetic factors of periodontal disease. Tobacco Induced Diseases, 2010; 8(1):4.

20. RIBEIRO M, CRUZ RC Jovens e o uso do narguilé: A saúde pode ser comprometida? Assobrafir Ciência, 2016; $7(1): 7-10$.

21. RYDER MI et al Personalized periodontal treatment for the tobacco- and alcohol-using patient. Periodontology 2000 , 2018; 78(1):30-46.

22. SALUM, AW; NETO, JBC; SALLUM; EJ Tabagismo e a doença periodontal. Revista Periodontia, 2007; 17(2):45-53.

23. SAPIENZA G, SCARINCI IC O isolamento em adolescentes que utilizam produtos derivados de tabaco. In: $12^{\circ}$ Congresso Nacional de Psicologia da Saúde, Lisboa. Actas do $12^{\circ}$ Congresso Nacional de Psicologia, ISPA Instituto Universitário, 2018.

24. SOUZA AB, et al. A obesidade como fator de risco para doença periodontal: revisão de literatura. Revista Dental Press Periodontia Implantologia, 2010; 4(4):30-39.

25. UNDERNER M, et al. Effects of smoking on periodontal disease. Revue des maladies respiratoires, 2009; 26(10):1057-1073.

26. VIEGAS, CAA Formas não habituais de uso do Tabaco. Jornal Brasileiro de Pneumologia, 2008; 34(2):1069-1073.

27. WHO report on the global tobacco epidemic, 2017: monitoring tobacco use and prevention policies. Geneva: World Health Organization; 2017. 